\title{
Adaptive optics fundus images of cone photoreceptors in the macula of patients with retinitis pigmentosa
}

This article was published in the following Dove Press journal:

Clinical Ophthalmology

22 January 2013

Number of times this article has been viewed

\section{Naoki Tojo \\ Tomoko Nakamura \\ Chiharu Fuchizawa \\ Toshihiko Oiwake \\ Atsushi Hayashi}

Department of Ophthalmology,

Graduate School of Medicine and

Pharmaceutical Sciences, University

of Toyama, Toyama, Japan
Correspondence: Atsushi Hayashi Department of Ophthalmology, Graduate School of Medicine and Pharmaceutical Sciences, University of Toyama, 2630 Sugitani, Toyama, 930-0194, Japan

$\mathrm{Tel}+8 \mathrm{I} 764347363$

Fax +8I 764345037

Email ganka@med.u-toyama.ac.jp
Background: The purpose of this study was to examine cone photoreceptors in the macula of patients with retinitis pigmentosa using an adaptive optics fundus camera and to investigate any correlations between cone photoreceptor density and findings on optical coherence tomography and fundus autofluorescence.

Methods: We examined two patients with typical retinitis pigmentosa who underwent ophthalmological examination, including measurement of visual acuity, and gathering of electroretinographic, optical coherence tomographic, fundus autofluorescent, and adaptive optics fundus images. The cone photoreceptors in the adaptive optics images of the two patients with retinitis pigmentosa and five healthy subjects were analyzed.

Results: An abnormal parafoveal ring of high-density fundus autofluorescence was observed in the macula in both patients. The border of the ring corresponded to the border of the external limiting membrane and the inner segment and outer segment line in the optical coherence tomographic images. Cone photoreceptors at the abnormal parafoveal ring were blurred and decreased in the adaptive optics images. The blurred area corresponded to the abnormal parafoveal ring in the fundus autofluorescence images. Cone densities were low at the blurred areas and at the nasal and temporal retina along a line from the fovea compared with those of healthy controls. The results for cone spacing and Voronoi domains in the macula corresponded with those for the cone densities.

Conclusion: Cone densities were heavily decreased in the macula, especially at the parafoveal ring on high-density fundus autofluorescence in both patients with retinitis pigmentosa. Adaptive optics images enabled us to observe in vivo changes in the cone photoreceptors of patients with retinitis pigmentosa, which corresponded to changes in the optical coherence tomographic and fundus autofluorescence images.

Keywords: adaptive optics fundus camera, cone photoreceptor, fundus autofluorescence, optical coherence tomography, retinitis pigmentosa

\section{Introduction}

Retinal degenerations constitute a group of inherited diseases that result in progressive loss of photoreceptors. In retinitis pigmentosa, cone dysfunction is secondary to rod degeneration and ultimately results in loss of fine visual acuity. ${ }^{1,2}$ Survival of photoreceptors seems to be closely correlated with visual functioning in patients with retinitis pigmentosa. ${ }^{2,3}$ Therefore, assessment of photoreceptors may be very important in evaluation of disease progression in patients with the disease.

The functioning of the retinal pigment epithelium can be visualized by fundus autofluorescence using a short wave length, which reflects lipofuscin accumulation in the retinal pigment epithelium or disrupted phagocytosis. ${ }^{4}$ Indeed, other studies have 
reported that an abnormal fundus autofluorescence ring is present in more than half of patients with retinitis pigmentosa examined, and that the length of the inner and outer segment (IS/OS) line determined by optical coherence tomography (OCT) is significantly correlated with the diameter of the fundus autofluorescence ring., ${ }^{2,5,6}$ In a study using new retinal imaging devices, such as spectral-domain OCT and adaptive optics scanning laser ophthalmoscopy, it was shown that patients with retinitis pigmentosa had abnormal cone spacing values, which were correlated with reduction in visual acuity and the amplitudes of multifocal electroretinograms. ${ }^{7}$

In the present study, we analyzed cone photoreceptors in the macula of patients with retinitis pigmentosa using an adaptive optics fundus camera, and examined the relationship between cone photoreceptor density and abnormal findings on fundus autofluorescence and OCT images.

\section{Materials and methods}

We examined two patients with retinitis pigmentosa at Toyama University Hospital. Each patient underwent a complete eye examination, including measurement of decimal best-corrected visual acuity and collection of scotopic and photopic full-field electroretinographic, OCT, fundus autofluorescent, and adaptive optics fundus camera images. OCT was performed using a threedimensional OCT-2000 system (Topcon Corp, Tokyo, Japan). Electroretinography was performed using an LE-4000 system (Tomey Corp, Aichi, Japan) according to the International Society for Clinical Electrophysiology of Vision standards. Fundus autofluorescent images were obtained with a scanning laser ophthalmoscope (F-10, Nidek Co, Aichi, Japan). A blue laser light (490 nm) was used for excitation and a wide bandpass filter with a cutoff at $521 \mathrm{~nm}$ was inserted in front of the detector for fundus autofluorescence. Adaptive optics fundus images were taken with a rtx-1 ${ }^{\text {TM }}$ flood-illuminated adaptive optics fundus camera (Imagine Eyes, Orsey, France). The rtx-1 has a resolution of $1.6 \mu \mathrm{m}$ with a $4 \times 4$ degree imaging field of view and an illumination wavelength of $850 \mathrm{~nm}$. The adaptive optics images taken with the rtx-1 were analyzed using the original cone analysis software created by the manufacturer.

To establish a healthy control group for purposes of comparison with the patients suffering from retinitis pigmentosa, we also included five healthy control eyes from five subjects. Adaptive optics images of the healthy control eyes were taken using the rtx-1 and analyzed with the same software. All procedures conformed to the tenets of the Declaration of Helsinki. The study protocol for the adaptive optics fundus camera was approved by the institutional review board of the University of Toyama. All subjects gave their written informed consent before participation in the study.

\section{Results \\ Case I}

Case 1 was a 31-year-old woman with best-corrected visual acuity of 0.9 in the right eye and 1.0 in the left eye. A color fundus photograph of the right eye showed fundus changes typical of retinitis pigmentosa, ie, black bone spicule pigmentation and depigmentation, narrowing of the retinal vessels, and retinal degeneration (Figure 1A). Scotopic and photopic full-field electroretinograms were undetectable in both eyes (data not shown). An abnormal parafoveal ring of high-density fundus autofluorescence was observed in the fundus autofluorescence image (arrows in Figure 1B), which indicated abnormal phagocytosis and concomitant lipofuscin accumulation in the retinal pigment epithelium. The fovea showed a normal dark area on fundus autofluorescence.

The OCT image of the macula of the right eye (Figure 1C) showed an irregular cone outer segment tips line and IS/OS line at the fovea. The normal dark macular fundus autofluorescence area corresponded to the area with the residual cone outer segment tips line and IS/OS line (Figure 1C and D).

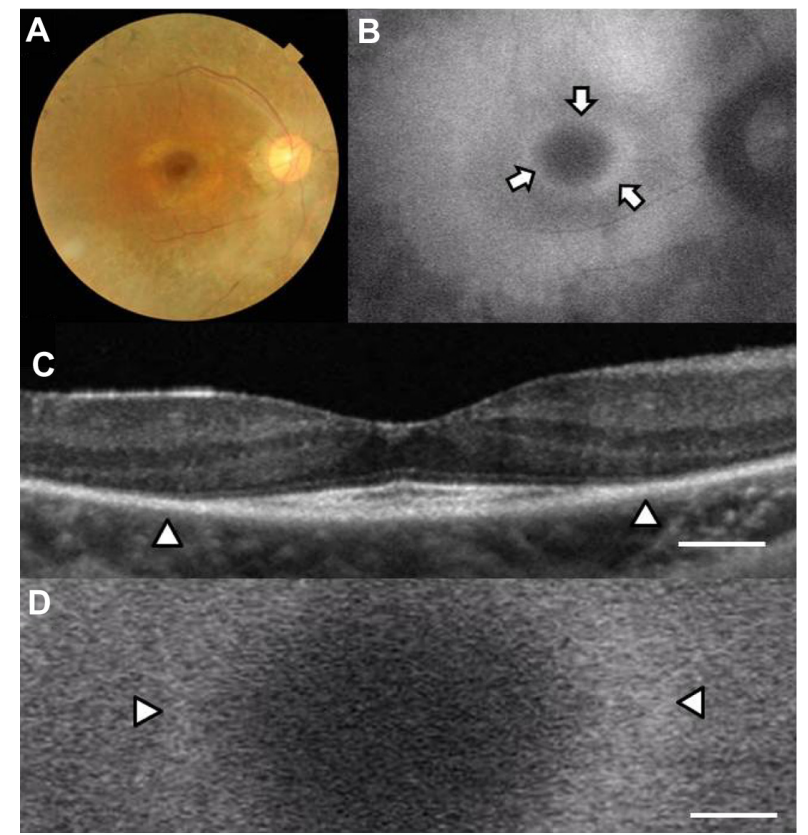

Figure I Fundus images of the right eye in case I. Color fundus photograph (A), short-wave length fundus autofluorescence photograph (B), spectral-domain optical coherence tomographic image (C), and magnified fundus autofluorescence photograph of the macula (D). Arrows show a parafoveal high-density fundus autofluorescence ring (B). Arrowheads show the border of the external limiting membrane and the inner segment and outer segment line (C) and parafoveal highdensity fundus autofluorescence ring (D). Bar $=200 \mu \mathrm{m}$ (C and D). 
The high-density autofluorescent ring in the fundus autofluorescence image corresponded to the border of the IS/OS line and the external limiting membrane in the OCT image (arrowheads in Figure 1C and D). However, it was difficult to determine whether the inner or outer border of the highdensity fundus autofluorescence ring corresponded to the termination of the IS/OS line and external limiting membrane in the OCT image.

Figure $2 \mathrm{~A}$ shows a panoramic image of cone photoreceptors in a $2.42 \times 3.16 \mathrm{~mm}$ area including the fovea of the right eye taken with the adaptive optics fundus camera. The number of cone photoreceptors was reduced in this panoramic adaptive optics image. A ring-shaped area with blurred cone mosaics and some patchy dark areas around the fovea was

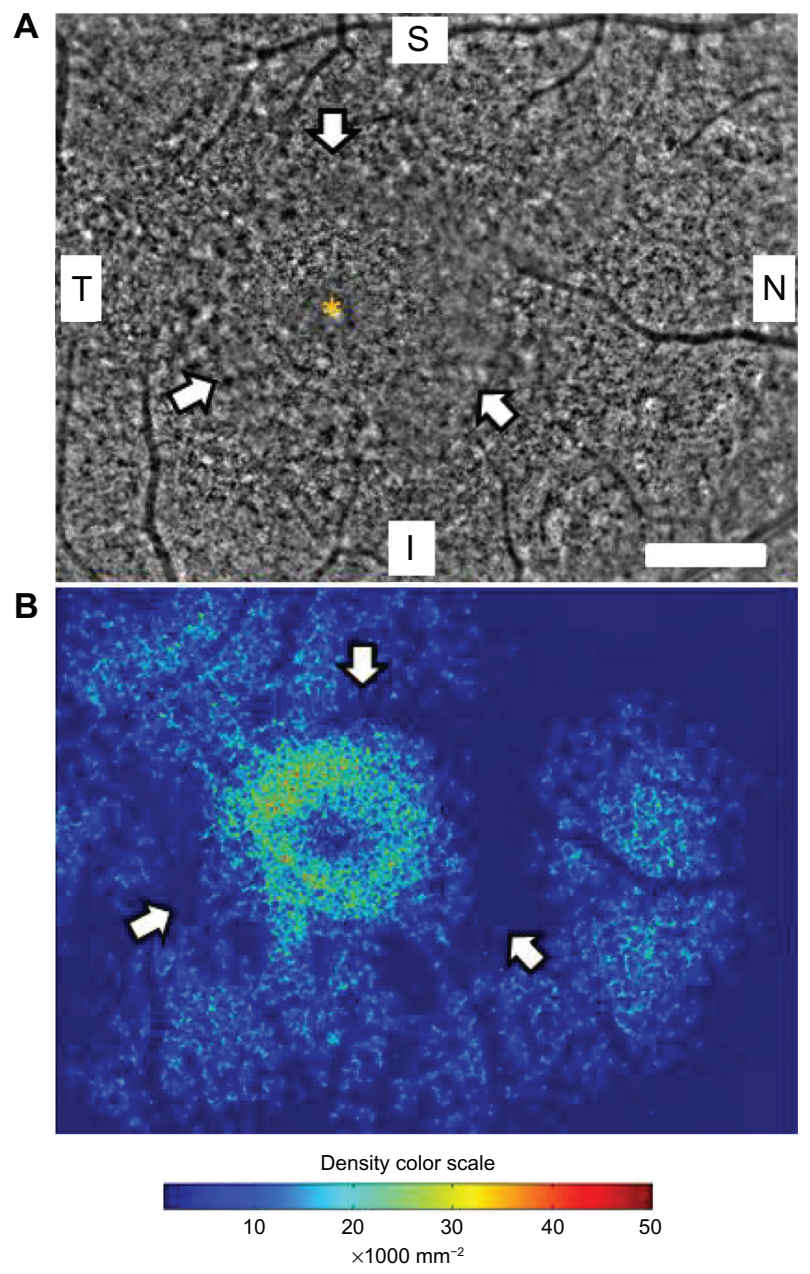

Figure 2 Panoramic adaptive optics fundus image $(\mathbf{A})$ and cone density map (B) of the right eye in case I. (A) Panoramic adaptive optics fundus image $2.42 \times 3.16 \mathrm{~mm}$ in area including the fovea (yellow asterisk). Arrows show a blurred ring area of cone mosaics which corresponds to the parafoveal high-density fundus autofluorescence ring. Bar $=200 \mu \mathrm{m}$ (A). (B) Panoramic cone density map of the same area as in (A). Notes: Arrows show a ring of low density area which corresponds to the blurred area in the adaptive optics image. Arrowheads show focal high density areas of cone photoreceptors. The color scale of the density is shown.

Abbreviations: S, superior; I, inferior; N, nasal; T, temporal. observed (arrows in Figure 2A). This ring-shaped blurred area corresponded to the high-density autofluorescent ring by fundus autofluorescence. Cone photoreceptors in the adaptive optics images were analyzed using the manufacturer's software. The cone density panorama map obtained from the image in Figure 2A is shown in Figure 2B. The blurred ring in Figure 2A shows a reduced density of cone photoreceptors (arrows in Figure 2B). In the region outside of the ring, areas of higher cone densities resembling islands were observed (arrowheads in Figure 2B). Adaptive optics fundus photographs were taken several times and photographs that were blurred by motion artifacts were not used.

Four $200 \times 200 \mu \mathrm{m}$ areas at the nasal or temporal retina were selected (avoiding large retinal vessels) and analyzed using the software (Figure 3). The nasal or temporal areas were selected at the blurred ring (N600 and T600) and outside of the ring (N1300 and T1100). The N600 and T600 distances were $600 \mu \mathrm{m}$ from the foveal center, and the N1300 and $\mathrm{T} 1100$ distances were $1300 \mu \mathrm{m}$ and $1100 \mu \mathrm{m}$ from the foveal center. The results for the three types of cone analyses at the four areas are shown in Figure 3, and the data are shown in Table 1 . The results of the cone analyses for the five healthy control eyes in the same four areas as examined in case 1 are also shown in Table 1. The control data are expressed as the mean \pm standard deviation for the five healthy control eyes. Figure 4 shows panoramic $(6.22 \times 0.96 \mathrm{~mm})$ and $0.2 \times 0.2 \mathrm{~mm}$ adaptive optics images in one of the five healthy control eyes obtained by rtx-1.

The densities of cone photoreceptors were lower at all four areas than those of the healthy control eyes (Table 1). As shown in Figure 2B, the cone densities at the blurred area of the ring (T600 and N600) were lower than the areas outside of the ring (T1100 and N1300), even though the OCT image showed loss of the IS/OS line and external limiting membrane. The results for the spacing and Voronoi domains were correlated with those of the densities.

\section{Case 2}

Case 2 was a 56-year-old woman with best-corrected visual acuity of 1.2 in both eyes. A color fundus photograph of the right eye showed fundus characteristics typical of retinitis pigmentosa, as also seen in case 1 (Figure 5A). Scotopic and photopic full-field electroretinograms were undetectable in both eyes (data not shown). A parafoveal ring with a high density of autofluorescence was observed by fundus autofluorescence (arrows in Figure 5B), but only the inner border of the temporal part of the ring was demarcated (arrowhead in Figure 5B). The IS/OS line and the external 

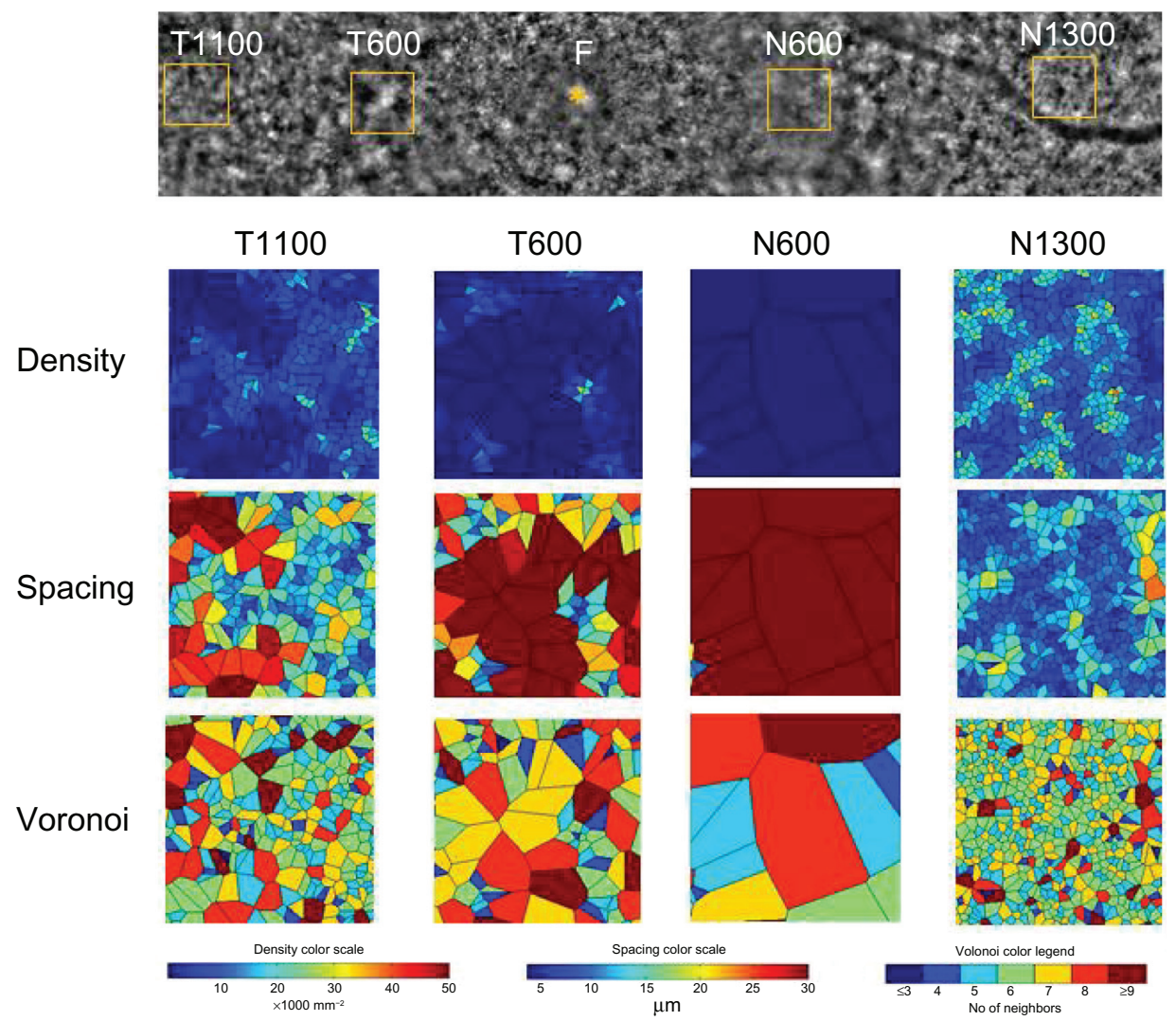

Figure 3 Cone analyses of the parafoveal areas in case 1.

Notes: Four areas of $0.2 \times 0.2 \mathrm{~mm}$ were selected for the nasal or temporal regions at the blurred ring (N600 and T600), and outside of the ring (NI300 and TII00). The N600, T600, NI300, and TII 00 distances were $600 \mu \mathrm{m}, 600 \mu \mathrm{m}, 1300 \mu \mathrm{m}$, and I I $00 \mu \mathrm{m}$ from the foveal center, respectively. Cone analysis maps of the density, spacing, and Voronoi domains are shown with color scales for each of the four areas.

limiting membrane were better preserved than in case 1 , and the border of the IS/OS line and the external limiting membrane was observed in the OCT image (arrowheads in Figure 6A). The nasal part of the high-density parafoveal fundus autofluorescence ring corresponded to the border of the IS/ OS line and the external limiting membrane (arrowheads in Figure 6B), but the temporal part of the ring was not clear. Figure $6 \mathrm{C}$ shows a panoramic image of cone photoreceptors in a $1.05 \times 5.88 \mathrm{~mm}$ area including the fovea of the right eye. The cone photoreceptors of the adaptive optics image were reduced in the macula, and the area with blurred cone mosaics and patchy dark spots corresponded to only the nasal part of the parafoveal ring (arrowheads in Figure 6C). The cone photoreceptors in the adaptive optics images were analyzed using the manufacturer's software. The cone density panoramic map (Figure 6D) shows that the area of the blurred cone mosaics in the nasal part of the ring has reduced cone densities (arrowheads in Figure 6D).

Four $200 \times 200 \mu \mathrm{m}$ areas at the nasal or temporal retina (Figure 7) were selected (avoiding large retinal vessels) and

Table I Results of cone analyses at four areas in the macula of the right eye in case I and healthy control eyes

\begin{tabular}{|c|c|c|c|c|}
\hline & TIIO0 & T600 & N600 & NI300 \\
\hline \multicolumn{5}{|l|}{ Density $\left(/ \mathrm{mm}^{2}\right)$} \\
\hline Case I & 5409 & 2053 & 124 & 12381 \\
\hline Healthy control $(n=5)$ & $|8| 18 \pm 196 \mid$ & $2469 I \pm 24 I I$ & $22822 \pm 3322$ & $15260 \pm 1851$ \\
\hline \multicolumn{5}{|l|}{ Spacing $(\mu \mathrm{m})$} \\
\hline Case I & 14.22 & 20.66 & 62.67 & 9.85 \\
\hline Healthy control $(n=5)$ & $8.23 \pm 0.44$ & $7.04 \pm 034$ & $7.34 \pm 0.53$ & $8.95 \pm 0.51$ \\
\hline \multicolumn{5}{|c|}{ Voronoi (\% of six-sided polygons) } \\
\hline Case I & 31.9 & 22.0 & 0 & 36.3 \\
\hline Healthy control $(n=5)$ & $51.6 \pm 5.0$ & $46.6 \pm 4.0$ & $44.0 \pm 5.1$ & $46.0 \pm 4.2$ \\
\hline
\end{tabular}

Note: Data for healthy controls are shown as the mean \pm standard deviation. 


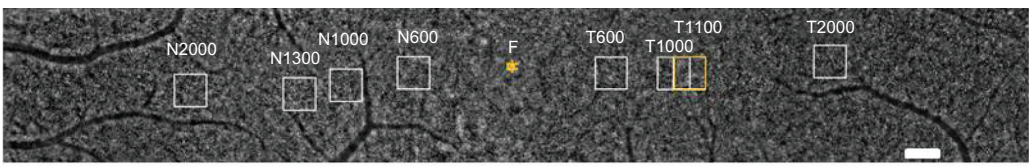

N600

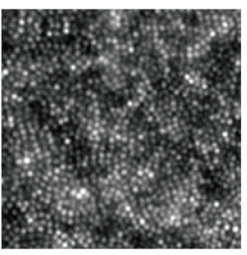

T600

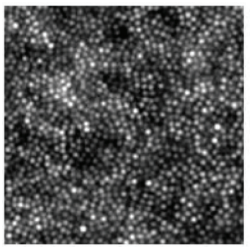

N1000

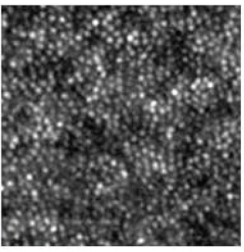

T1000

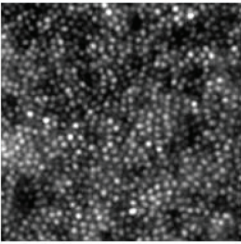

N1300

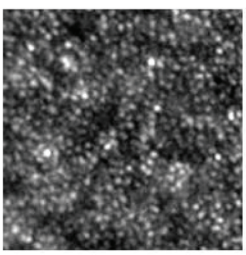

T1100

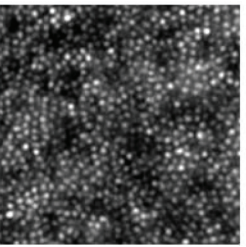

N2000

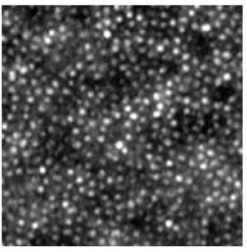

T2000

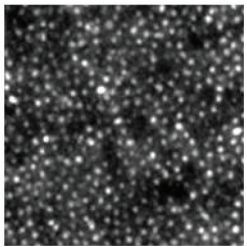

Figure 4 Panoramic and focal adaptive optics images of the macular area in one eye for the healthy controls.

Notes: A panoramic adaptive optics image showing a $6.22 \times 0.96 \mathrm{~mm}$ area including the foveal center (yellow asterisk, F). Bar $=200 \mu \mathrm{m}$. Eight $0.2 \times 0.2 \mathrm{~mm}$ areas were selected at the nasal or temporal retina and each adaptive optics image is shown. The N600, NI000, NI300, N2000, T600, TI000, TII00, and T2000 distances were 600 $\mu$ m, $1000 \mu \mathrm{m}, 1300 \mu \mathrm{m}, 2000 \mu \mathrm{m}, 600 \mu \mathrm{m}, 1000 \mu \mathrm{m}, 1 \mathrm{I} 00 \mu \mathrm{m}$, and $2000 \mu \mathrm{m}$ from the foveal center, respectively.

analyzed using the manufacturer's software. The nasal area was selected at the inside of the blurred ring (N1000) and at the border of the ring (N2000). The temporal areas (T1000 and T2000) were selected at the same distance as the two nasal areas. The N1000 and T1000 distances were $1000 \mu \mathrm{m}$ from the foveal center, and those of N2000 and T2000 were $2000 \mu \mathrm{m}$ from the foveal center. The results for the three types of cone analyses at the four areas are shown in Figure 7, and the data are shown in Table 2. The mean densities of the cone photoreceptors were lower at T1000, N1000, and N2000 than for the healthy controls (Table 2). Because the temporal part of the fundus autofluorescence ring was not clear, the number of cone photoreceptors remained the same as that in the healthy controls at T2000 in case 2 . The results for the spacing and Voronoi domains were correlated with those of the densities.
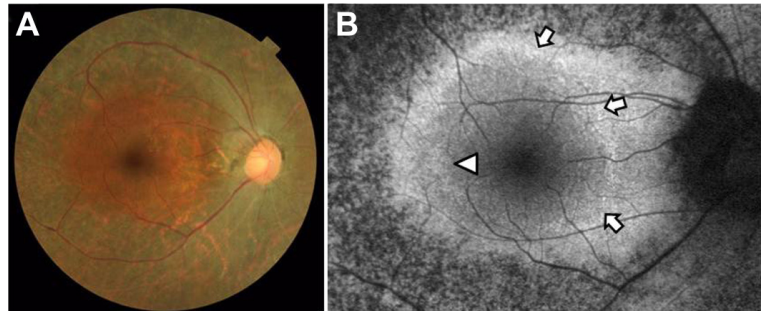

Figure 5 Color fundus photograph (A) and short-wavelength fundus autofluorescence photograph (B) of the right eye in case 2. Arrows show an incomplete parafoveal ring of high-density fundus autofluorescence (B). The arrowhead shows the inner border of the high-density fundus autofluorescence ring (B).

\section{Discussion}

Retinitis pigmentosa causes progressive degeneration of photoreceptors and the retinal pigment epithelium. These changes were observed as an abnormal parafoveal ring of high-density or low-density fundus autofluorescence at

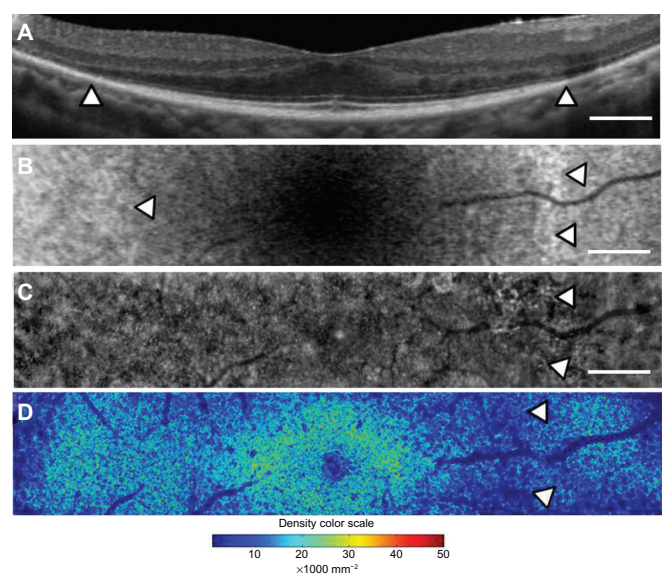

Figure 6 Macular images of OCT (A), fundus autofluorescence (B), adaptive optics (C), and the panoramic cone density map (D) of the right eye in case 2 . The OCT image shows the border of the inner segment and outer segment line and the external limiting membrane at the arrowheads (A). Magnified fundus autofluorescence image of the macula shows part of the high-density fundus autofluorescence ring at the nasal retina [arrowheads in (B)]. The temporal inner border of the ring is indicated by an arrowhead (B). The adaptive optics panoramic image of a $5.9 \times 1.1 \mathrm{~mm}$ area appears as a blurred region with some dark patchy areas at the nasal side of the high-density fundus autofluorescence ring (arrowheads in C).A panoramic cone density map of the same area as in (C) shows low densities at the nasal ring [arrowheads in (D)]. Bar $=200 \mu \mathrm{m}$ (A-C).

Note: The color scale of the density is shown.

Abbreviation: OCT, optical coherence tomography. 

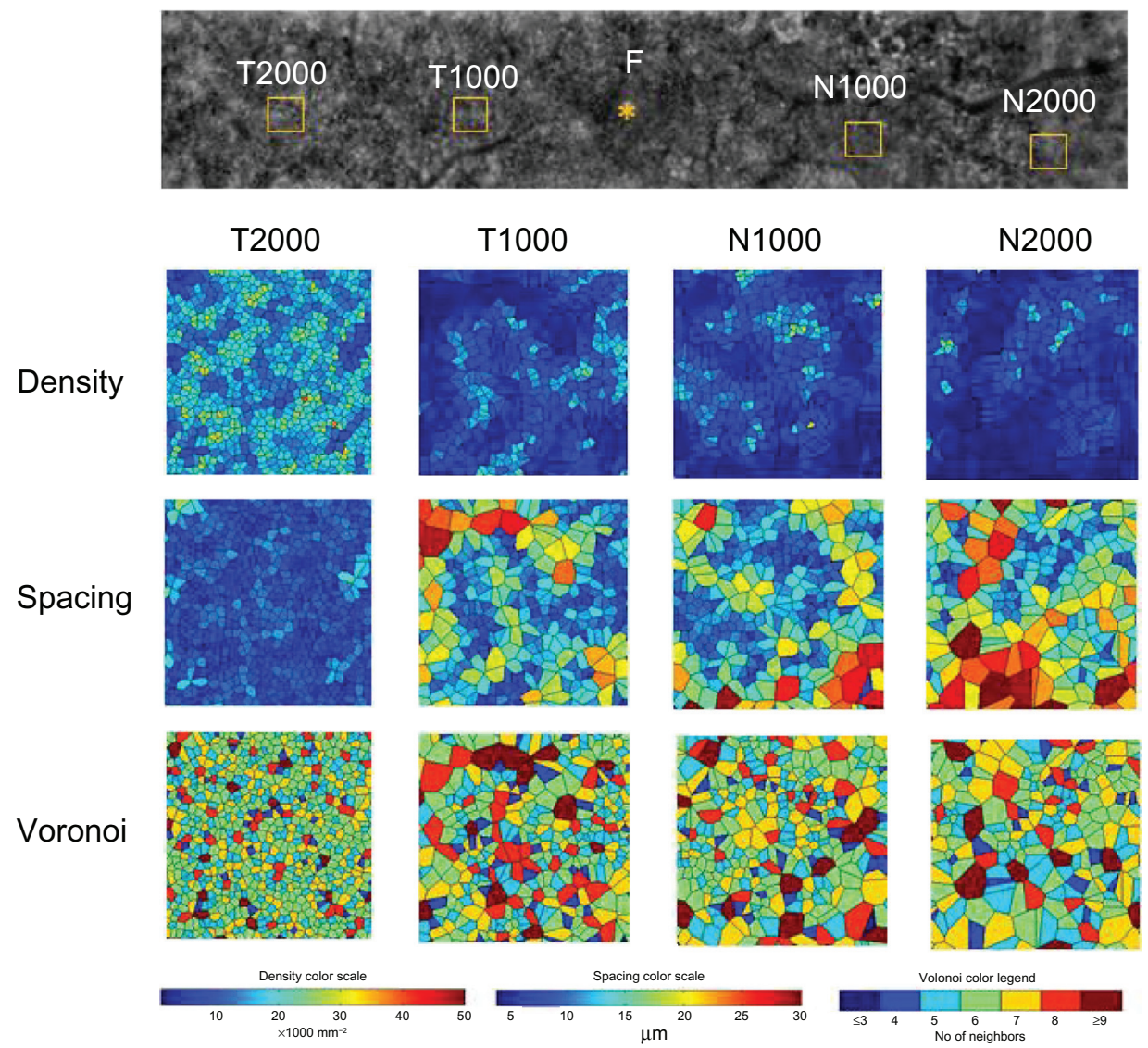

Figure 7 Cone analyses of the parafoveal areas in case 2.

Notes: Four areas were selected at the nasal areas inside of the blurred ring (NI000) and at the ring (N2000) and the temporal areas at the same distance from the two nasal areas (TI000 and T2000). The NI000, TI000, N2000, and T2000 distances were $1000 \mu \mathrm{m}, 1000 \mu \mathrm{m}, 2000 \mu \mathrm{m}$, and $2000 \mu \mathrm{m}$ from the foveal center, respectively. Cone analysis maps of the density, spacing, and Voronoi domains are shown with color scales for each of the four areas.

Abbreviation: $F$, foveal center.

the macula by fundus autofluorescence imaging in patients with retinitis pigmentosa. ${ }^{2,3,8}$ In fact, previous studies have reported that an abnormal parafoveal ring of high-density fundus autofluorescence was observed in more than half of patients with retinitis pigmentosa studied. ${ }^{2,5}$ The highdensity parafoveal fundus autofluorescence ring area represented a transition between the functional and dysfunctional retina, and the diameter and area of the high-density fundus autofluorescence ring were significantly correlated with retinal sensitivity and lengths of the IS/OS line and cone outer segment tips line in patients with retinitis pigmentosa. ${ }^{2,5,6}$

In the present study, we used an adaptive optics fundus camera to observe cone photoreceptors in vivo and showed that the blurred areas of cone mosaics in adaptive optics images corresponded to the high-density fundus autofluorescence ring in the fundus autofluorescence images and

Table 2 Results of cone analyses at four areas in the macula of the right eye in case 2 and healthy control eyes

\begin{tabular}{|c|c|c|c|c|}
\hline & T2000 & T1000 & N1000 & N2000 \\
\hline \multicolumn{5}{|l|}{ Density $\left(/ \mathrm{mm}^{2}\right)$} \\
\hline Case 2 & 16267 & 7371 & 7180 & 4820 \\
\hline Healthy control $(n=5)$ & $13822 \pm 1395$ & $|967| \pm 2794$ & $16979 \pm 1853$ & $12886 \pm 1213$ \\
\hline \multicolumn{5}{|l|}{ Spacing $(\mu \mathrm{m})$} \\
\hline Case 2 & 8.67 & 12.06 & 12.45 & 15.06 \\
\hline Healthy control $(n=5)$ & $9.40 \pm 0.47$ & $8.10 \pm 0.43$ & $8.49 \pm 0.46$ & $9.7 \mathrm{I} \pm 0.38$ \\
\hline \multicolumn{5}{|c|}{ Voronoi (\% of six-sided polygons) } \\
\hline Case 2 & 40.9 & 30.8 & 34.6 & 34.0 \\
\hline Healthy control $(n=5)$ & $46.0 \pm 2.4$ & $51.7 \pm 3.6$ & $44.9 \pm 2.4$ & $41.9 \pm 3.2$ \\
\hline
\end{tabular}

Note: Data for healthy controls are shown as the mean \pm standard deviation. 
to the border of the external limiting membrane and the IS/OS line in OCT images. Because a previous histological study $^{9}$ found that the foveal center had high cone densities of $100,000-324,000 / \mathrm{mm}^{2}$, we concluded that the adaptive optics fundus camera did not have sufficient resolution to distinguish between individual cones at the foveal center. Therefore, cone analyses at the foveal center were not performed. Loss of the external limiting membrane, IS/OS line, and cone outer segment tips line in the OCT images may represent loss of cell bodies of photoreceptors, which appeared as dark patchy areas without cone mosaics or blurred reflections in adaptive optics images in case 1 . The area with a detectable external limiting membrane in the OCT images showed decreased cone mosaics and blurred reflections in adaptive optics images as well as relatively normal autofluorescence in fundus autofluorescence images. However, as shown in Tables 1 and 2, the areas outside of the high density fundus autofluorescence ring (T1100 and N1300 in case 1 and T2000 in case 2) showed higher densities than those at the ring (T600 and N600 in case 1 and $\mathrm{T} 1000$ in case 2). We compared the results of cone analyses for the five healthy control eyes and two eyes of patients with retinitis pigmentosa using the same manufacturer's software. Although the high-density fundus autofluorescence ring corresponded to the area with low cone densities, the nasal area of case 1 (N1300) and the temporal area of case 2 (T2000) in the region outside of the ring showed relatively normal cone densities that corresponded to the cone density maps in both cases. These results suggest that degeneration of photoreceptors might occur unevenly in retinitis pigmentosa, even though a high-density fundus autofluorescence ring was observed.

Histopathological studies of retinitis pigmentosa have shown that dysfunction of the rods precedes that of the cones and that the shortening of the rod outer segments and photoreceptor cell death occur at the mid-peripheral retina. ${ }^{1,10}$ Photoreceptors in all types of retinitis pigmentosa were decreased at the parafoveal areas by up to $1.5 \mathrm{~mm}$ compared with normal retinas. ${ }^{8}$ After the death of photoreceptors, the retinal pigment epithelium migrates into the inner retina, and the processes of Mueller cells become hypertrophied. ${ }^{1}$

In both of the cases reported here, the cone densities were decreased in a wide area of the temporal and nasal retina. Cone spacing and Voronoi analyses showed similar abnormalities. In the cone density maps of both cases, more cones were observed in nasal areas outside of the ring (N1300 in case 1) and in the temporal area of the parafoveal high-density fundus autofluorescence ring (T2000 in case 2), suggesting that the rate of loss of photoreceptors might be different in the nasal area from that in the temporal area. The patchy dark areas around the fovea in the adaptive optics images may represent a loss of photoreceptors and proliferation of retinal pigment epithelium.

In this study we demonstrated that cone photoreceptors were decreased in the macula of patients with retinitis pigmentosa and especially at the abnormal high-density fundus autofluorescence ring using an adaptive optics fundus camera. The abnormal findings on OCT and fundus autofluorescence images were correlated with the decrease in cone photoreceptors shown by adaptive optics images. The results suggest that adaptive optics images may have advantages for visualizing the horizontal distribution of cone photoreceptors and increasing our understanding of the pathophysiology of retinitis pigmentosa. Further studies using adaptive optics fundus imaging are needed to evaluate successive changes in the cone photoreceptors of patients with retinitis pigmentosa over the long term.

\section{Disclosure}

The authors report no conflicts of interest in this work.

\section{References}

1. Milam AH, Li ZY, Fariss RN. Histopathology of the human retina in retinitis pigmentosa. Prog Retin Eye Res. 1998;17:175-205.

2. Mitamura Y, Mitamura-Aizawa S, Nagasawa T, Katome T, Eguchi H, Naito T. Diagnostic imaging in patients with retinitis pigmentosa. J Med Invest. 2012;59:1-11.

3. Wakabayashi T, Sawa M, Gomi F, Tsujikawa M. Correlation of fundus autofluorescence with photoreceptor morphology and functional changes in eyes with retinitis pigmentosa. Acta Ophthalmol. 2010;88: 177-183.

4. Kellner U, Kellner S, Weber BHF, Fiebig B, Weinitz S, Ruether K. Lipofuscin- and melanin-related fundus autofluorescence visualize different retinal pigment epithelial alterations in patients with retinitis pigmentosa. Eye. 2009;23:1349-1359.

5. Murakami T, Akimoto M, Ooto S, et al. Association between abnormal autofluorescence and photoreceptor disorganization in retinitis pigmentosa. Am J Ophthalmol. 2008;145:687-694.

6. Aizawa S, Mitamura Y, Hagiwara A, Sugawara T, Yamamoto S. Changes of fundus autofluorescence, photoreceptor inner and outer segment junction line, and visual function in patients with retinitis pigmentosa. Clin Experiment Ophthalmol. 2010;38:597-604.

7. Duncan JL, Zhang Y, Gandhi J, et al. High-resolution imaging with adaptive optics in patients with inherited retinal degeneration. Invest Ophthalmol Vis Sci. 2007;48:3283-3291.

8. Lenassi E, Troeger E, Wilke R, Hawlina M. Correlation between macular morphology and sensitivity in patients with retinitis pigmentosa and hyperautofluorescent ring. Invest Ophthalmol Vis Sci. 2012;53: 47-52.

9. Curcio CA, Sloan KR, Kalina RE, Hendrickson AE. Human photoreceptor topography. J Comp Neurol. 1990;292:497-523.

10. Stone JL, Barlow WE, Humayun MS, de Juan E Jr, Milam AH. Morphometric analysis of macular photoreceptors and ganglion cells in retinas with retinitis pigmentosa. Arch Ophthalmol. 1992;110: 1634-1639. 


\section{Publish your work in this journal}

Clinical Ophthalmology is an international, peer-reviewed journal covering all subspecialties within ophthalmology. Key topics include: Optometry; Visual science; Pharmacology and drug therapy in eye diseases; Basic Sciences; Primary and Secondary eye care; Patient Safety and Quality of Care Improvements. This journal is indexed on

Submit your manuscript here: http://www.dovepress.com/clinical-ophthalmology-journal
PubMed Central and CAS, and is the official journal of The Society of Clinical Ophthalmology (SCO). The manuscript management system is completely online and includes a very quick and fair peer-review system, which is all easy to use. Visit http://www.dovepress.com/ testimonials.php to read real quotes from published authors. 